\title{
Prevention of perinatal group B streptococcal infection: Management strategies
}

$\mathrm{G}_{\mathrm{n}}^{\mathrm{r}}$ roup B streptococcus (GBS) infects two to three per 1000 newborns in the United States with a mortality of $20 \%$ to $30 \%$ (1); although specific Canadian data are not known, they are likely similar. As many as $10 \%$ to $30 \%$ of pregnant women are colonized with GBS in the vagina or rectum $(2,3), 1 \%$ to $2 \%$ of their newborns developing early onset disease (4). Chemoprophylaxis of the newborn infant has limited effectiveness $(4,5)$. A recent meta-analysis of several studies have demonstrated the efficacy of intrapartum penicillin chemoprophylaxis for the prevention of early onset GBS infection (6), although another meta-analysis has questioned whether there is yet demonstrated proof that intrapartum chemoprophylaxis significantly decreases neonatal mortality from GBS (7). Despite the apparent effectiveness of intrapartum penicillin chemoprophylaxis, controversy persists regarding the identification of the population that would most benefit from it.

As many as 19 different strategies for screening and chemoprophylaxis to prevent early onset GBS infection have been identified (8), although most have not been tested for efficacy. Based on a study demonstrating the efficacy of early third-trimester screening for GBS and intrapartum chemoprophylaxis (9), the American Academy of Pediatrics (AAP) recommended universal screening for GBS colonization at 26 to 28 weeks' gestation and intrapartum treatment for GBS-colonized women with specific risk factors (10). The American College of Obstetricians and Gynecologists (ACOG) preferred an approach that did not include screening cultures of women at any time during pregnancy. Based on a study from Atlanta, Georgia (11), they recommended chemoprophylaxis of women with specific risk factors (12). The Canadian Paediatric Society (CPS) and the Society of Obstetricians and Gynaecologists of Canada (SOGC) emphasized that the critical issue is the iden-

All material presented in Pediatric Infectious Disease Notes has been reviewed and approved by the chairperson, Canadian Paediatric Society's Board of directors and representative members of the Canadian Paediatric Society Committee on Infectious Diseases and Immunization

Correspondence: Infectious Diseases and Immunization Committee, Canadian Paediatric Society, 2204 Walkley Road, Suite 100, Ottawa, Ontario K1G 4G8. Telephone 613-526-9397. fax 613-526-3332 tification of at risk deliveries and suggested that either the approach of the AAP or the ACOG was acceptable (13).

Recently, the Centers for Disease Control and Prevention (CDC), Atlanta, Georgia, reviewed GBS prevention strategies and published new guidelines for the prevention of early onset GBS disease (14). The new guidelines are based on a concern that early third-trimester screening is insufficiently predictive of colonization at the time of delivery and on a discomfort with withholding chemoprophylaxis to GBS-positive mothers in the absence of risk factors. The $\mathrm{CDC}$ recommended two alternative strategies, both of which are believed to be effective but which have not been tested in prospective clinical trials. The proposed first strategy is a nonscreening approach similar to the strategy previously recommended by the ACOG. The second strategy proposed by the CDC involves prenatal GBS cultures at 35 to 37 weeks' gestation. Intrapartum chemoprophylaxis is recommended for all preterm deliveries and for all women who are GBS carriers, regardless of the presence or absence of risk factors. As seen in Table 1 from the CDC guidelines (14), this latter strategy is estimated to prevent the most cases of neonatal GBS infection but requires treating the greatest proportion of mothers. The strategy of screening at 26 to 28 weeks' gestation and treating colonized women with risk factors requires treatment of fewer mothers but is estimated to be the least effective (predicting only $67 \%$ of women who are GBS-positive at term [3]) and is not recommended by the CDC (14). An algorithm for pregnant women (modified from CDC guidelines) is presented in Figure 1.

Methods for obtaining and processing specimens for culture of GBS are outlined in previous guidelines (10,12-14). Difficulties with isolating GBS may be partially dependent on the broth used. Although CDC guidelines suggest Todd-Hewitt broth may be supplemented with either colistin and nalidixic acid or gentamicin and nalidixic acid, use of the latter requires blood supplementation (15); gentamicin may inhibit many GBS stains (16). It is also essential that swabs be placed directly in selective growth media because placement in standard growth media with subsequent transfer to selective media substantially reduces yield of GBS (17).

The management of a woman in premature labour whose labour stops is problematic. The CDC recommends that if the culture is negative and premature labour stops, antimicrobial 
TABLE 1: Estimated impact of several strategies for the use of intrapartum antimicrobial prophylaxis (IAP) against early onset group B streptococcal (GBS) disease in a hypothetical population

\section{Prevention strategy}

Prenatal culture at 35 to 37 weeks' gestation; IAP for preterm deliveries and all GBS carriers

No prenatal cultures; IAP for all women with intrapartum risk factors (eg, fever, prolonged rupture of membranes, more than 37 weeks' gestation)

Prenatal cultures at 26 to 28 weeks' gestation; IAP for GBS carriers who develop risk factors

*Percentage was estimated for a hypothetical population; proportion of deliveries among women who had intrapartum risk factors was $24.7 \%$ (18); ${ }^{+}$Percentage was estimated for a hypothetical population; proportion of deliveries among GBS positive women who had intrapartum risk factors was $4.6 \%$ (18)

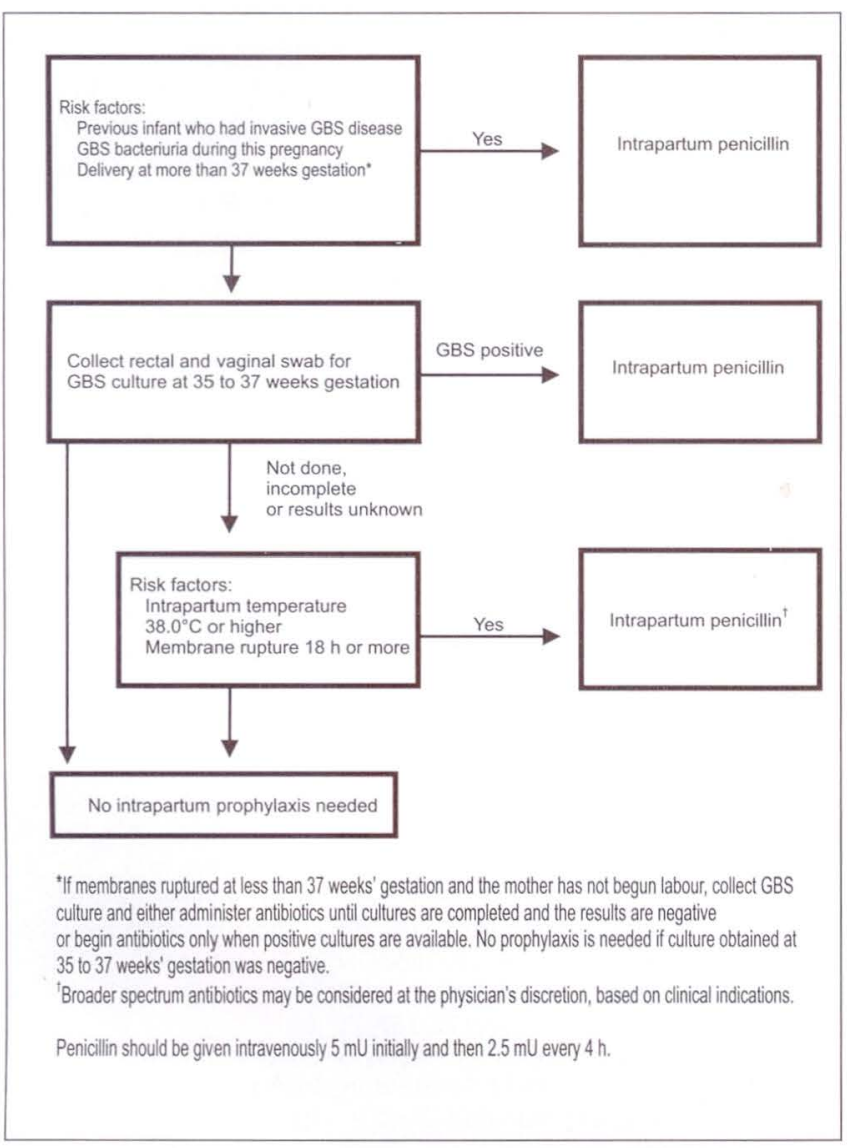

Figure 1) Algorithm for prevention of early onset group B streptococcal (GBS) disease in neonates, using prenatal screening at 35 to 37 weeks' gestation

therapy can be discontinued and no further therapy is necessary (14). If the culture is positive for GBS, intrapartum chemoprophylaxis should be provided whenever labour resumes.

Management of the newborn whose mother had received intrapartum chemoprophylaxis is empirical; an approach was suggested in the CPS/SOGC guidelines. A simpler algorithm, similarly untested, is included in the CDC guidelines and has been modified as presented in Figure 2. These recommendations consider the presence of signs of infection, the gesta-

\section{Proportion of early onset} GBS disease prevented (\%) 86.0

50.7
Proportion of deliveries receiving IAP (\%)

26.7

$18.3^{*}$

3.4

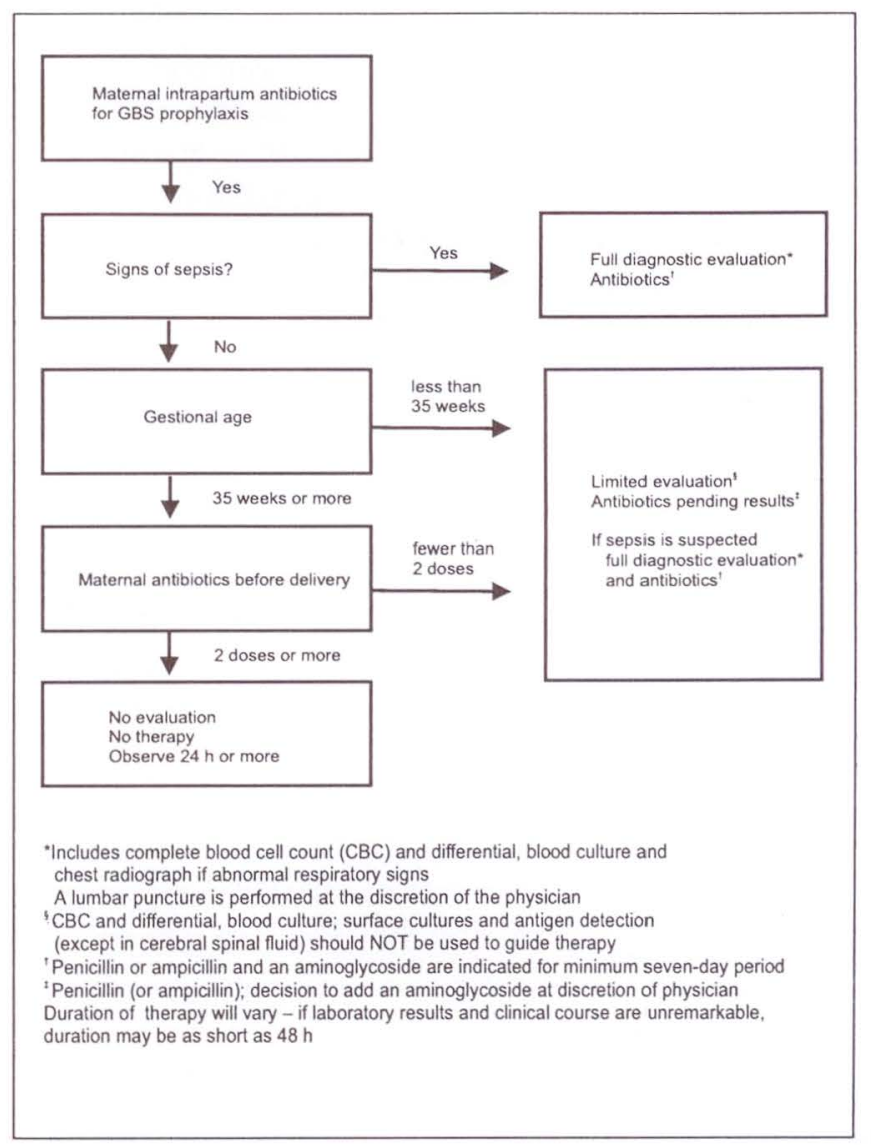

Figure 2) Approach to the neonate with indicated maternal intrapartum antibiotic prophylaxis for group B streptococcus (CBS) infection

tional age of the newborn and the adequacy of the maternal chemoprophylaxis. The observation period for the term babies who have received adequate intrapartum antibiotic prophylaxis has been reduced to $24 \mathrm{~h}$ in hospital in accordance with Canadian guidelines (17).

With the publication of the CDC guidelines, the practitioner is now faced with three different strategies, all of which are estimated to be cost effective (20-22). Each has advantages and disadvantages. The AAP recommendation treats the fewest pregnancies but is estimated to prevent the lowest propor- 
tion of GBS disease. However, in practice, the proportion of pregnancies treated may be higher because of an unwillingness by women and their physicians to ignore a positive GBS culture, even in the absence of other risk factors. The ACOG strategy is simplest in that it requires no prenatal cultures and prevents more GBS disease, but it requires that substantially more women receive chemoprophylaxis. The CDC late pregnancy cultures and treatment of all colonized women is estimated to prevent the most cases but requires the treatment of even more women. In view of the increasing prevalence of antimicrobial resistance worldwide, the prospect of antimicrobial use in over $25 \%$ of all pregnancies raises concerns (23). In addition, the CDC statement refers to the potential for 10 maternal deaths per year from anaphylaxis $(0.001 \%)$ with another $0.7 \%$ to $10 \%$ women having less severe reactions (14).

Based on the limitations encountered with the screening strategy of cultures at 26 to 28 weeks' gestation, we believe that either strategy published by the CDC is acceptable in Canada; there are still insufficient data to recommend a single preferred strategy. If the practitioner uses the screening strategy, it is essential that specimens be obtained and processed in an appropriate manner. The algorithm in Figure 2, based on

\section{REFERENCES}

1. Fischer G, Horton RE, Edelman R. Summary of the National Institute of Health Workshop on group B streptococcal infection. J Infect Dis 1983;148:163-6.

2. Regan JA, Klebanoff MA, Nugent RP. Vaginal infection and prematurity study group. The epidemiology of group B streptococcal colonization in pregnancy. Obstet Gynecol 1991;77:604-10.

3. Boyer KM, Gadzola CA, Kelly PD, Burg LI, Gotoff SP. Selective intrapartum chemoprophylaxis of neonatal group B streptococcal early-onset disease. II. Predictive value of perinatal cultures. J Infect Dis 1983;148:802-9.

4. Pyati SP, Pildes RS, Jacobs NM, et al. Penicillin in infants weighing $2 \mathrm{~kg}$ or less with early-onset group B streptococcal disease. N Engl J Med 1983;308:1383-9.

5. Baker CJ, Edwards MS. Group B streptococcal infections: Perinatal impact and prevention methods. Ann NY Acad Sci 1988;549:193-202.

6. Allen UD, Navas L, King SM. Effectiveness of intrapartum penicillin prophylaxis in preventing early-onset group B streptococcal infections: Results of a meta-analysis. Can Med Assoc J 1993:149:1659-65.

7. Johlsson A, Myhr TL. Intrapartum chemoprophylaxis of perinatal group B streptococcal infections: A critical review of randomized controlled trials. Am J Obstet Gynecol 1994;170:910-917.

8. Rouse DJ, Goldenberg RL, Cliver SP, Cutter GR, Mennemeyer ST, Fargason CA. Strategies for the prevention of early-onset neonatal group B streptococcal sepsis: A decision analysis. Obstet Gynecol 1994;83:483-94.

9. Boyer KM, Gotoff SP. Prevention of early-onset neonatal group B streptococcal disease with selective intrapartum chemoprophylaxis. N Engl J Med 1986;314:1665-9.

10. Committee on Infectious Diseases and Committee on Fetus and Newborn. Guidelines for prevention of group B streptococcal (GBS) infection by chemoprophylaxis. Pediatrics 1992;90:775-8.

11. Schuchat A, Oxtoby M, Cochi S, et al. Population-based risk factors for neonatal group B streptococcal disease: Results of a cohort study in metropolitan Atlanta. J Infect Dis 1990;162:672-7.

12. Group B streptococcal infections in pregnancy. ACOG Tech Bull 1992;170:1-5. the CDC guidelines, is a reasonable alternative to the CPS and SOGC guidelines for neonatal management.

It must be emphasized that no strategy can prevent all cases of GBS disease and that disease may occur despite intrapartum chemoprophylaxis. Further data are required on the epidemiology of GBS colonization and disease in Canada, the effectiveness of these alternative intrapartum antibiotic chemoprophylaxis strategies and the impact of widespread use of antimicrobial agents in pregnancy on the bacterial microflora. However, it is our belief that a standardized approach whereby all at risk pregnancies are-identified and managed by one of these recommended strategies will allow the appropriate assessment upon which further refinements of the guidelines can be based.

Nestor N Demianczuk MD FRCSC, Department of Obstetrics and Gynecology, University of Alberta, Edmonton, Alberta; Scott A Halperin MD FRCPC, Department of Pediatrics, Dalhousie University, Halifax, Nova Scotia;

Douglas D McMillan MD FRCPC, Department of Pediatrics, University of Calgary, Calgary, Alberta

13. Society of Obstetricians and Gynecologists of Canada and the Canadian Paediatric Society. National consensus statement on the prevention of early-onset group B streptococcal infections in the newborn. J Soc Obstet Gynaecol Can 1994;16:2271-8.

14. US Department of Health and Human Services, Public Health Service, Centers for Disease Control and Prevention. Prevention of perinatal group B streptococcal disease: A public health perspective. MMWR 1996;45:1-24.

15. Fenton LJ, Harper MN. Evaluation of colistin and nalidixic acid in Todd-Hewitt broth for selective isolation of group B streptococci. J Clin Microbiol 1979;9:167-9.

16. Gray BM, Pass MA, Dillon HC. Laboratory and field evaluation for selective media for isolation of group B streptococci. J Clin Microbiol 1979;9:466-70.

17. Silver HM, Struminsky J. A comparison of the yield of positive group B streptococcus culture with direct inoculation in selective growth media versus primary inoculation in transport medium followed by delayed inoculation in selective growth media. Am J Obstet Gynecol 1996;175:155-7.

18. Canadian Paediatric Society, Society of Obstetricians and Gynaecologists of Canada. Facilitating discharge home following a normal term birth. Paediatr Child Health 1996;1:165-9.

19. Boyer KM, Gotoff SP. Strategies for chemoprophylaxis of GBS early-onset infections. Antibiot Chemother 1985;35:267-80.

20. Mohle-Boetani JC, Schuchat A, Plikaytis BD, Smith JD, Broome $\mathrm{CV}$. Comparison of prevention strategies for neonatal group B streptococcal infection: A population-based economic analysis. JAMA 1993;270:1442-8.

21. Strickland DM, Yeomans ER, Hankins GDV. Cost-effectiveness of intrapartum screening and treatment for maternal group B streptococci colonization. Am J Obstet Gynecol 1990;163:4-8.

22. Yancey MK, Duff P. An analysis of the cost-effectiveness of selected protocols for the prevention of neonatal group B streptococcal infection. Obstet Gynecol 1994;83:367-71.

23. McDuffie RS, McGregor JA, Gibbs RS. Adverse perinatal outcome and resistance enterobacteriaceae after antibiotic use for premature rupture of the membranes and group B streptococcus carriage. Obstet Gynecol 1993;82:487-9. 


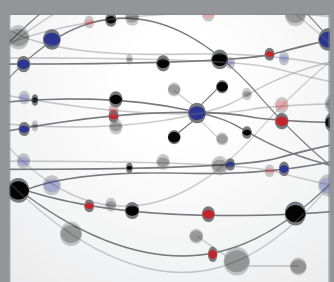

The Scientific World Journal
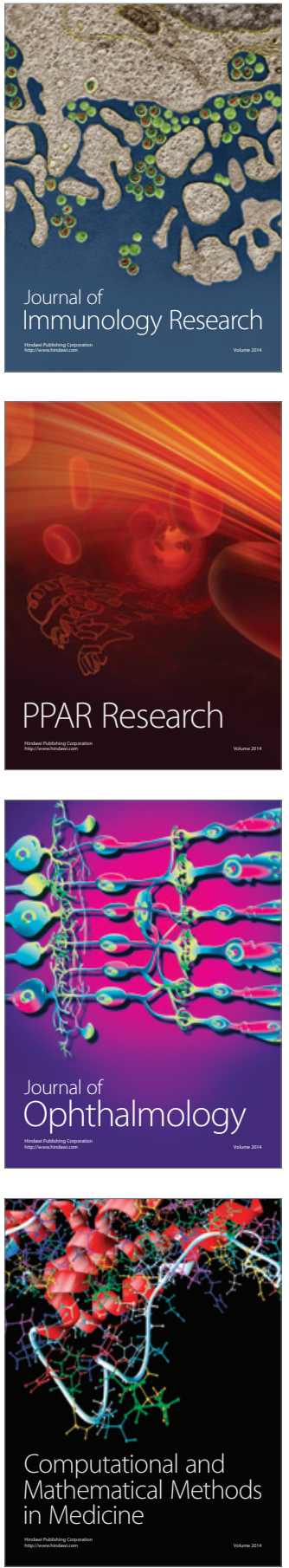

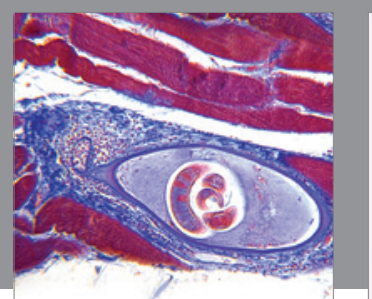

Gastroenterology Research and Practice

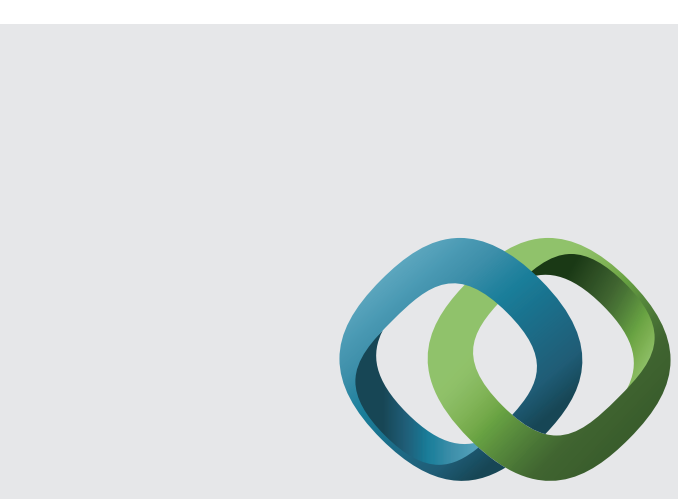

\section{Hindawi}

Submit your manuscripts at

http://www.hindawi.com
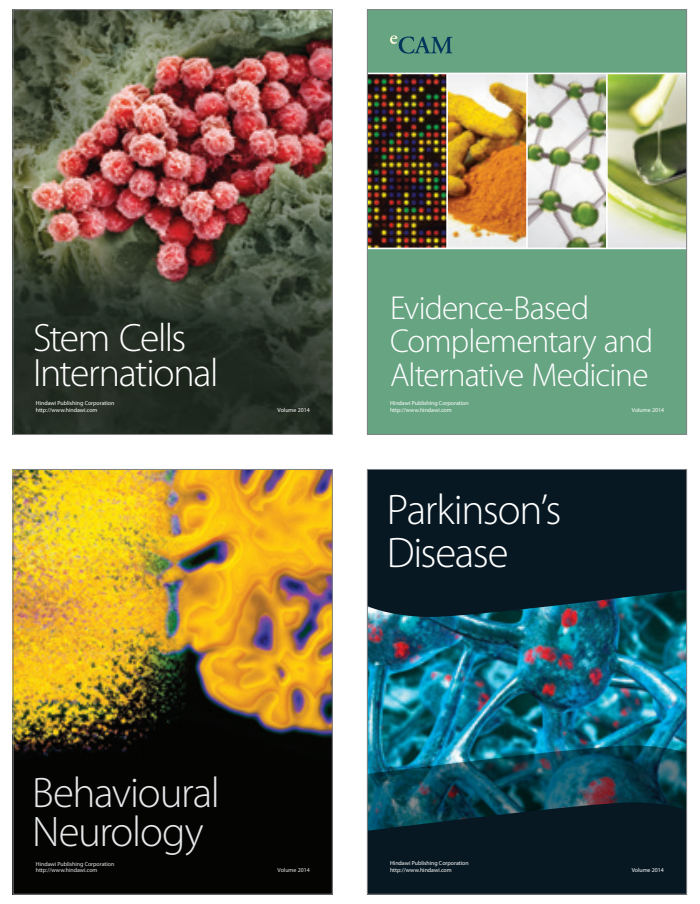
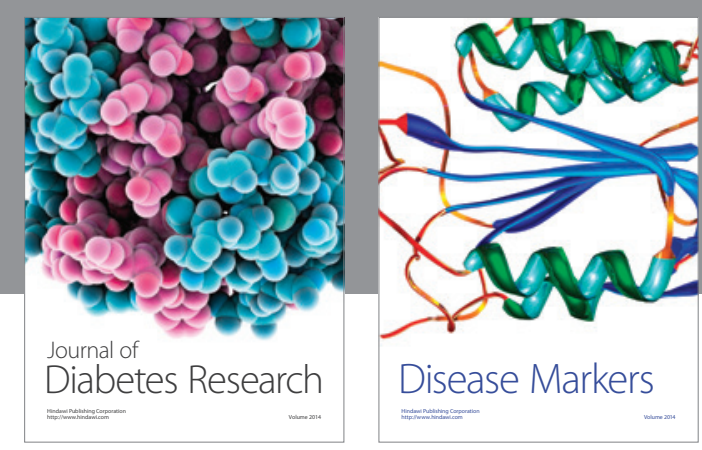

Disease Markers
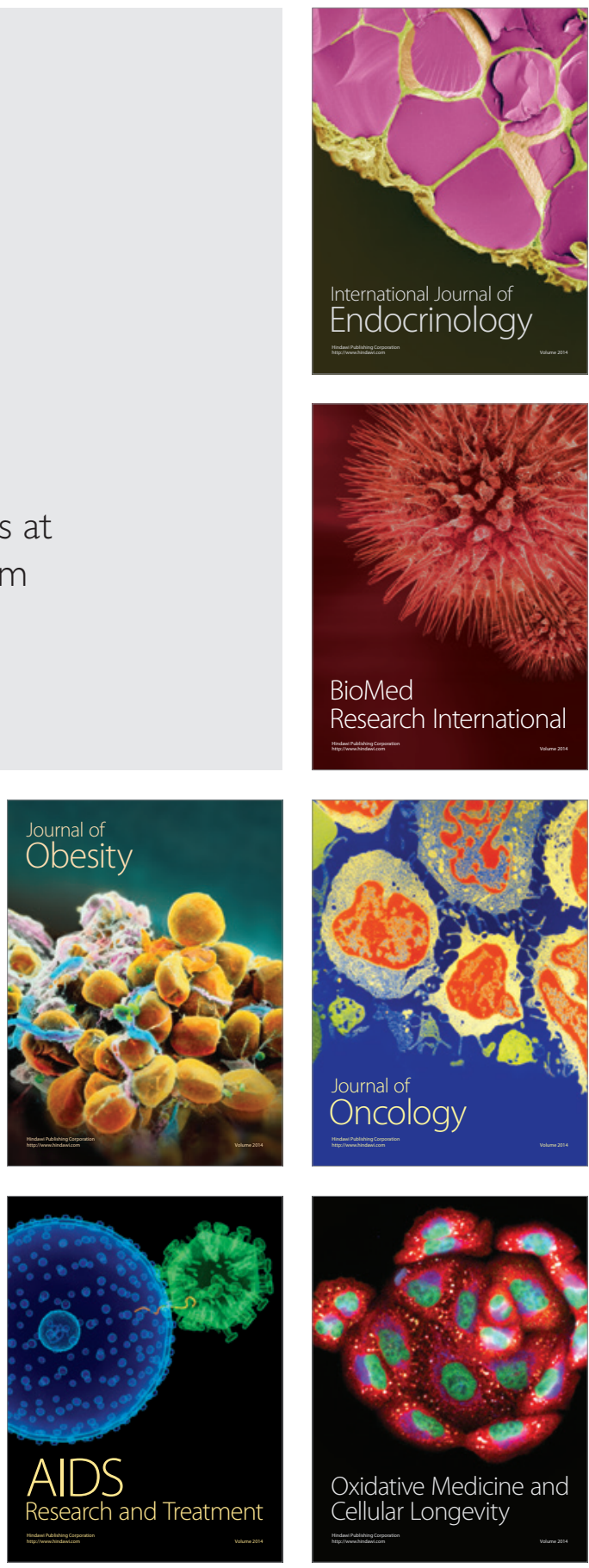